\title{
Multi-level differential network analysis of COPD exacerbations
}

\author{
Guillaume Noell ${ }^{1,2,12}$, Borja G. Cosío (10 ${ }^{1,3,12}$, Rosa Faner ${ }^{1,2,12}$, Eduard Monsó ${ }^{1,4}$ \\ German Peces-Barba ', Alfredo de Diego ${ }^{6}$, Cristobal Esteban ${ }^{7}$, Joaquim Gea ${ }^{1,8}$,' \\ Robert Rodriguez-Roisin ${ }^{1,2,9}$, Marian Garcia-Nuñez ${ }^{1,4}$, Francisco Pozo- \\ Rodriguez ${ }^{1,10}$, Susana G. Kalko ${ }^{2}$ and Alvar Agustí1,2,9,11
}

\begin{abstract}
Affiliations: ${ }^{1}$ CIBER Enfermedades Respiratorias (CIBERES), Instituto de Salud Carlos III, Barcelona, Spain. ${ }^{2}$ Institut d'investigacions Biomèdiques August Pi i Sunyer (IDIBAPS), Barcelona, Spain. ${ }^{3}$ Servei Pneumologia, Hospital Universitari Son Espases-IdISBa, Mallorca, Spain. ${ }^{4}$ Servei Pneumologia, Hospital Parc Taulí, Sabadell, Spain. ${ }^{5}$ Servicio Neumología, Fundación Jimenez Díaz, Madrid, Spain. 'Servicio Neumología, Hospital Universitario La Fe, Valencia, Spain. ${ }^{7}$ Servicio Neumología, Hospital Galdakao, Bizkaia, Spain. ${ }^{8}$ Servei de Pneumologia, Hospital del Mar - IMIM. DCEXS, Universitat Pompeu Fabra, Barcelona, Spain. ${ }^{9}$ Departament de Medicina, Universitat de Barcelona, Barcelona, Spain. ${ }^{10} \mathrm{Hospital}$ Universitario 12 de Octubre, Madrid, Spain. ${ }^{11}$ Respiratory Institute, Hospital Clinic, University of Barcelona, Barcelona, Spain. ${ }^{12}$ Joint lead authors.
\end{abstract}

Correspondence: Alvar Agustí, Respiratory Institute, Hospital Clinic, Villarroel 170, 08036 Barcelona, Spain. E-mail: AAGUSTIAclinic.cat

\section{@ERSpublications}

This is the first study to investigate COPD exacerbations using multi-level differential network analysis http://ow.ly/uYlW30eMpwR

Cite this article as: Noell G, Cosío BG, Faner R, et al. Multi-level differential network analysis of COPD exacerbations. Eur Respir J 2017; 50: 1700075 [https://doi.org/10.1183/13993003.00075-2017].

ABSTRACT Patients with chronic obstructive pulmonary disease (COPD) often suffer episodes of exacerbation (ECOPD) that impact negatively the course of their disease. ECOPD are heterogeneous events of unclear pathobiology and non-specific diagnosis. Network analysis is a novel research approach that can help unravelling complex biological systems. We hypothesised that the comparison of multi-level (i.e., clinical, physiological, biological, imaging and microbiological) correlation networks determined during ECOPD and convalescence can yield novel patho-biologic information.

In this proof-of-concept study we included 86 patients hospitalised because of ECOPD in a multicentre study in Spain. Patients were extensively characterised both during the first $72 \mathrm{~h}$ of hospitalisation and during clinical stability, at least 3 months after hospital discharge.

We found that 1) episodes of ECOPD are characterised by disruption of the network correlation observed during convalescence; and 2) a panel of biomarkers that include increased levels of dyspnoea, circulating neutrophils and C-reactive protein (CRP) has a high predictive value for ECOPD diagnosis (AUC 0.97).

We conclude that ECOPD 1) are characterised by disruption of network homeokinesis that exists during convalescence; and 2) can be identified objectively by using a panel of three biomarkers (dyspnoea, circulating neutrophils and CRP levels) frequently determined in clinical practice.

This article has supplementary material available from erj.ersjournals.com

Received: Jan 132017 | Accepted after revision: June 262017

This study is registered at www.clinicaltrials.gov as NCT01750658.

Support statement: Supported, in part, by Fondo de Investigación Sanitaria, Instituto Carlos III (PI 05/1463 and PI 15/ 00799), CIBERES, an unrestricted grant from GlaxoSmithKline, a $\mathrm{PhD}$ scholar grant from the Catalan Government (FI-2016) and CERCA Programme/Generalitat de Catalunya. Funding information for this article has been deposited with the Crossref Funder Registry.

Conflict of interest: Disclosures can be found alongside this article at erj.ersjournals.com

Copyright CERS 2017 


\section{Introduction}

Patients with chronic obstructive pulmonary disease (COPD) often suffer episodes of exacerbation (ECOPD) that impact negatively their health status and prognosis [1]. The pathogenesis of these episodes is not entirely understood, but it is presumed complex and heterogeneous [2-4]. Their diagnosis relies mostly on symptom perception by the patient [5] and their prevention and treatment is, by and large, empiric [1].

Network analysis is an integrative research strategy well suited for the investigation of heterogeneous and complex diseases [6,7] such as COPD [8-14]. We hypothesised that multi-level differential network analysis (MLDNA), a novel analytical method that involves the comparison of clinical, physiological, biological, imaging and microbiological (i.e. multi-level) correlation networks determined during ECOPD and clinical stability, can provide new insights into the pathobiology and diagnosis of ECOPD [15, 16]. Accordingly, in this proof-of-concept study we used MLDNA, for the first time to our knowledge, to 1) compare the multi-level network structure determined during ECOPD and convalescence; and 2) identify a panel of specific ECOPD biomarkers.

\section{Methods}

Methods are described in detail in the supplementary material.

\section{Study design and ethics}

This observational, prospective proof-of-concept study was carried out in seven tertiary referral hospitals in Spain (www.clinicaltrials.gov: NCT01750658). Patients were recruited and studied during the first $72 \mathrm{~h}$ of hospitalisation because of ECOPD, and investigated again during convalescence, at least 3 months after hospital discharge. The Institutional Review Boards of participating institutions approved the study, and participants gave their informed consent.

\section{Patients}

All patients were older than 45 years, current or former smokers (>10 pack-years) and had COPD (and ECOPD) according to the Global Initiative for Chronic Obstructive Lung Disease criteria [1]. In order to homogenise the studied population as much as possible, pneumonia on chest radiography, the presence of severe comorbidity driving the clinical presentation of the patient and/or need of (invasive or noninvasive) mechanical ventilation were exclusion criteria. We initially attempted to recruit patients who had not received oral steroids and/or antibiotic treatment in the community before hospitalisation. Yet, this strategy limited recruitment a lot, so we decided to adopt a more pragmatic design and exclude patients who received oral steroids before hospitalisation (with a potential rapid anti-inflammatory effect) but not those who may have received antibiotics (which may take longer to affect microbiological results). A total of 14 patients (16\%) were included in the analysis despite they received antibiotic treatment in the community before hospitalisation.

\section{Measurements}

Clinical, functional, biological, microbiological and imaging variables were recorded following standard procedures, as detailed in the supplementary material.

\section{Data analysis}

\section{Descriptive statistics}

Because many variables were non-normally distributed, the results are presented as median (and 95\% confidence intervals) or proportions. Likewise, because not all measurements were available in all patients in both visits, to maximise the potential of available information, the results at ECOPD and convalescence were compared using pairwise statistics (paired Wilcoxon or Chi-squared tests for continuous and discrete variables, respectively). Participants with missing data were discarded on a per-variable basis, such that no value imputation was required. We used false discovery rates (FDRs) to account for multiple comparisons [17]. All analyses were performed using R [18].

\section{Multi-level correlation networks}

We built multi-level correlation (Spearman) networks that integrate quantitative and qualitative clinical, functional, biological, microbiological and imaging variables (independently for ECOPD and convalescence) using R [18], and we graphed them with Cytoscape [19].

\section{Module finding}

We used the fast-greedy community algorithm to identify network modules on the basis of their module modularity $(\mathrm{MM})$ score, so those with more dense internal connections and fewer external links get higher MM scores [20, 21]. 
Differential network analysis

To compare multilevel correlation networks at ECOPD and convalescence we 1) nominally contrasted the variables and modules identified under both clinical circumstances; 2) estimated the mean "density" of networks determined at ECOPD and convalescence by comparing (Wilcoxon test) the number of nodes, and the average number of edges per node (node degree, $k$ ) during ECOPD and convalescence [6]; and, 3) used Monte Carlo permutation tests [22] to identify those Spearman correlations that were significantly different between ECOPD and convalescence.

\section{ECOPD biomarkers}

We defined as "outliers" at ECOPD those values below or above the 5th or 95th percentiles, respectively, of the same variable at convalescence, and we identified those ECOPD variables with a significant (bootstrapping FDR <0.05) number of outliers. To identify potential ECOPD biomarkers, we calculated receiver operating characteristic (ROC) curves considering all values determined at ECOPD and convalescence and excluding missing data on a per-variable basis.

\section{Results}

We studied 86 patients at ECOPD (mean \pm SD age of $67 \pm 9$ years). As shown in figure 1, 19 patients were lost for follow-up, so we could study 67 of them at convalescence. Table 1 presents the main characteristics of participants at both time points.

\section{Observations at ECOPD}

Besides the expected observations during ECOPD (dyspnoea, tachypnoea, tachycardia, respiratory failure) some other salient findings were (table 1): 1) elevated blood glucose levels, likely to be in relation to the generalised use of systemic steroids in the management of ECOPD [1, 23]; 2) echography identified the presence of pulmonary hypertension in $21.2 \%$ of patients and right chamber enlargement in $19.1 \%$, but no patient suffered heart failure with low ejection fraction; 3) computed tomography (CT) emphysema was present in $56.7 \%$ of patients, bronchiectasis in $17.5 \%$ and, interestingly, alveolar infiltrates (not seen in chest radiography films) in $23.8 \%$. Pulmonary embolism was found in $1.5 \%$ of individuals; and 4) in patients producing spontaneous sputum $(77.9 \%)$, bacterial culture was positive for potential pathogenic microorganisms (PPMs) in $19.4 \%$ of them, whereas viruses were detected by a positive sputum virus in $30.9 \%$. A total of $37.8 \%$ of patients were positive for sputum PPMs and/or viruses (table 1). More detailed microbiologic information can be found in the supplementary material.

\section{Changes at convalescence}

The main changes from ECOPD to convalescence (highlighted in bold type in table 1) included 1) improved dyspnoea; 2) reduced heart and respiratory rate; 3) reduced serum levels of glucose and urea; 4) improved pulmonary gas exchange without significant changes in spirometric variables; 5) reduced total leukocyte count, with lower circulating neutrophils and higher lymphocyte and eosinophil proportions; 6) reduced concentration of acute phase reactants (C-reactive protein (CRP) and fibrinogen) with increased levels of serum amyloid A (SAA). Other systemic inflammatory markers did not change significantly or changed marginally; and, finally, 7) neither bacterial load, viral load nor inflammatory markers changed significantly.

\section{Multi-level differential network analysis}

Figure 2 shows the correlation networks determined at ECOPD and convalescence, and table 2 their quantitative comparison. The main observations were 1) the number of nodes at ECOPD and

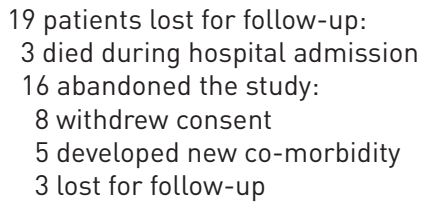


TABLE 1 Clinical, physiologic, imaging, biological and microbiological data determined during exacerbation of chronic obstructive pulmonary disease (ECOPD) and convalescence

$\frac{\text { ECOPD }}{\mathrm{n} \quad \text { Median }(95 \% \mathrm{CI}) \text { or } \mathrm{n}(\%)} \frac{\text { Convalescence }}{\mathrm{n} \quad \text { Median }(95 \% \mathrm{CI}) \text { or } \mathrm{n}(\%)}$

\begin{tabular}{|c|c|c|c|c|c|c|}
\hline \multicolumn{7}{|l|}{ Vital constants } \\
\hline Heart rate $\min ^{-1}$ & 83 & $89(72-112.5)$ & 64 & $83(69-100.5)$ & 64 & 0.004 \\
\hline Respiratory rate $\min ^{-1}$ & 82 & $22(15-30)$ & 64 & $20(16-24.5)$ & 64 & 0.000 \\
\hline Dyspnoea; MMRC scale & 79 & $5(2-5)$ & 63 & $3(1.5-5)$ & 59 & 0.010 \\
\hline Body temperature ${ }^{\circ} \mathrm{C}$ & 81 & $36.5(35.85-37.25)$ & 63 & $36(35.55-36.6)$ & 63 & 0.000 \\
\hline \multicolumn{7}{|l|}{ Biochemistry } \\
\hline Urea $\mathrm{mg} \cdot \mathrm{dL}^{-1}$ & 81 & $42(27-77)$ & 65 & $33(19-53)$ & 63 & 0.000 \\
\hline Haemoglobin $\mathrm{g} \cdot \mathrm{dL}^{-1}$ & 82 & $14.8(12.4-17.1)$ & 65 & $14.7(12.25-16.8)$ & 64 & 0.724 \\
\hline Erythrocyte sedimentation rate $\mathrm{mm} \cdot \mathrm{h}^{-1}$ & 64 & $21(3-67.51)$ & 55 & $12(2-46.5)$ & 52 & 0.002 \\
\hline \multicolumn{7}{|l|}{ Lung physiology } \\
\hline FVC \% reference & 86 & $71.5(51.5-106.5)$ & 63 & $75(56-109)$ & 63 & 1.000 \\
\hline $\mathrm{FEV}_{1} \%$ reference & 86 & $44.2(25.5-77)$ & 63 & $46(28-81.79)$ & 63 & 0.687 \\
\hline FEV $1 / F V C \%$ & 86 & $47.9(31.05-64.5)$ & 63 & $49.4(31-65.5)$ & 63 & 0.882 \\
\hline DLco \% reference & 72 & $56(31.5-88)$ & 52 & $54(30-84)$ & 50 & 0.035 \\
\hline Kco \% reference & 71 & $73(37.99-111)$ & 57 & $79(51-103)$ & 53 & 0.474 \\
\hline $\mathrm{PaO}_{2} \mathrm{mmHg}$ & 82 & $55.4(41.05-74.1)$ & 64 & $66.2(52.5-91.1)$ & 63 & 0.000 \\
\hline PAFI & 76 & $254.3(173.55-322.38)$ & 64 & $315.2(247.17-438.57)$ & 57 & 0.000 \\
\hline $\mathrm{PaCO}_{2} \mathrm{mmHg}$ & 82 & $43.5(32.95-69.76)$ & 64 & $42.7(34.9-53.35)$ & 63 & 0.605 \\
\hline Arterial pH & 82 & $7.4(7.33-7.47)$ & 63 & $7.4(7.37-7.46)$ & 62 & 0.946 \\
\hline $6 \mathrm{MWD} \mathrm{m}$ & 67 & $435(238.5-536.5)$ & 44 & $443.5(274-580)$ & 42 & 0.280 \\
\hline \multicolumn{7}{|l|}{ Cardiovascular physiology } \\
\hline Creatinine phosphokinase $U \cdot \mathrm{L}^{-1}$ & 70 & $68(30.5-238.51)$ & 58 & $69.5(39-150)$ & 56 & 0.914 \\
\hline Fibrinogen $\mathrm{mg} \cdot \mathrm{dL}^{-1}$ & 74 & $497(307.5-760)$ & 58 & $405(307.5-574.51)$ & 57 & 0.000 \\
\hline Pro-BNP $\mathrm{pg} \cdot \mathrm{mL}^{-1}$ & 85 & $0.4(0.1-0.78)$ & 67 & $0.4(0.04-1.06)$ & 67 & 0.882 \\
\hline Troponine I \% detected above $0.05 \mu \mathrm{g} \cdot \mathrm{L}^{-1}$ & 73 & $6(8.2 \%)$ & 60 & $3(5.0 \%)$ & & 0.872 \\
\hline \multicolumn{7}{|l|}{ Echography } \\
\hline \multicolumn{7}{|l|}{ Lung inflammation (sputum) } \\
\hline TAS mM & 84 & $0.3(0.05-1.29)$ & 67 & $0.2(0-1.26)$ & 67 & 0.977 \\
\hline $\mathrm{IL}-8 \mathrm{pg} \cdot \mathrm{mL}^{-1}$ & 84 & 2146.7 (791.68-2575.22) & 67 & $2128.3(25.91-2580.18)$ & 67 & 0.458 \\
\hline $\mathrm{IL}-1 \beta \mathrm{pg} \cdot \mathrm{mL}^{-1}$ & 84 & 621.5 (73.33-3194.87) & 67 & $504.9(1.39-2861.79)$ & 67 & 0.490 \\
\hline $\mathrm{IL}-6 \mathrm{pg} \cdot \mathrm{mL}^{-1}$ & 84 & $43.2(3.05-572.87)$ & 67 & $39.8(3.05-758.15)$ & 67 & 0.392 \\
\hline $\mathrm{TNF}-\alpha \mathrm{pg} \cdot \mathrm{mL}^{-1}$ & 83 & $14.3(0.52-552.37)$ & 67 & $6.2(0.52-345.42)$ & 67 & 0.450 \\
\hline TGF- $\beta$ pg $\cdot \mathrm{mL}^{-1}$ & 84 & $0.2(0.02-2.89)$ & 67 & $0.3(0-3.79)$ & 67 & 0.392 \\
\hline $\mathrm{TNF} \mathrm{RS} \mathrm{pg} \cdot \mathrm{mL}^{-1}$ & 84 & $1.3(0.04-11.78)$ & 67 & $2(0.01-21.61)$ & 67 & 0.621 \\
\hline SAA $\mathrm{pg} \cdot \mathrm{mL}^{-1}$ & 84 & $3.3(0.38-16.72)$ & 67 & $2.4(0.09-14.37)$ & 67 & 0.392 \\
\hline \multicolumn{7}{|l|}{ Systemic inflammation } \\
\hline Leukocytes $\times 10^{3} \mu \mathrm{L}^{-1}$ & 82 & $10.9(6.35-22.59)$ & 65 & $8.1(5.97-12.78)$ & 64 & 0.000 \\
\hline Neutrophils \% & 72 & $88.2(52.35-94.15)$ & 58 & $64.8(38.55-75.7)$ & 58 & 0.000 \\
\hline Lymphocytes \% & 82 & $7.4(3.75-20.25)$ & 65 & $22.1(14-38.4)$ & 64 & 0.000 \\
\hline Eosinophils \% & 53 & $0.3(0-2.45)$ & 64 & $2.4(1.05-7.8)$ & 39 & 0.000 \\
\hline$\%$ of patients with eosinophils $>2 \%$ & 53 & $3(5.7 \%)$ & 64 & $35(54.7 \%)$ & 53 & 0.000 \\
\hline $\mathrm{C}$-reactive protein $\mathrm{mg} \cdot \mathrm{L}^{-1}$ & 86 & $3.6(0.43-16.82)$ & 66 & $0.5(0.09-6.1)$ & 66 & 0.000 \\
\hline Total antioxidant status $\mathrm{mM}$ & 86 & $1.5(0.98-2.41)$ & 67 & $1.6(0.79-2.48)$ & 67 & 0.724 \\
\hline
\end{tabular}


TABLE 1 Continued

\begin{tabular}{|c|c|c|c|c|c|c|}
\hline & \multicolumn{2}{|r|}{ ECOPD } & \multicolumn{2}{|r|}{ Convalescence } & \multirow[t]{2}{*}{ Pairwise $n$} & \multirow[t]{2}{*}{ FDR p-value } \\
\hline & $\mathbf{n}$ & Median $(95 \% \mathrm{Cl})$ or $\mathrm{n}(\%)$ & $\mathbf{n}$ & Median $(95 \% \mathrm{CI})$ or $\mathrm{n}(\%)$ & & \\
\hline $\mathrm{IL}-8 \mathrm{pg} \cdot \mathrm{mL}^{-1}$ & 84 & $1(0.26-4.37)$ & 67 & $1.3(0.35-4.53)$ & 67 & 0.128 \\
\hline $\mathrm{IL}-6 \mathrm{pg} \cdot \mathrm{mL}^{-1}$ & 80 & $0.3(0.3-7.41)$ & 67 & $0.3(0.3-8.81)$ & 67 & 0.290 \\
\hline $\mathrm{TNF}-\alpha \mathrm{pg} \cdot \mathrm{mL}^{-1}$ & 79 & $0.5(0.51-1.89)$ & 67 & $1.3(0.51-2.49)$ & 67 & 0.000 \\
\hline Procalcitonin $\mathrm{mg} \cdot \mathrm{L}^{-1}$ & 85 & $0.4(0.09-0.93)$ & 67 & $0.4(0.03-0.86)$ & 67 & 0.605 \\
\hline SAA $p g \cdot \mathrm{mL}^{-1}$ & 84 & $0.8(0.13-4.11)$ & 67 & $1.4(0.13-7.64)$ & 67 & 0.002 \\
\hline \multicolumn{7}{|l|}{ Microbiology } \\
\hline Spontaneous sputum production & 86 & $67(77.9 \%)$ & 67 & $45(67.2 \%)$ & & 0.513 \\
\hline Positive sputum bacteria (culture) & 67 & $13(19.4 \%)$ & 45 & $12(26.7 \%)$ & & 0.848 \\
\hline Positive sputum virus (PCR) & 55 & $17(30.9 \%)$ & 27 & $3(11.1 \%)$ & & 0.394 \\
\hline Positive bacteria (culture) and/or virus (PCR) & 74 & $28(37.8 \%)$ & 59 & $14(23.7 \%)$ & & 0.753 \\
\hline Parainfluenza seroconversion & 43 & $7(16.3 \%)$ & ND & ND & & \\
\hline RSV seroconversion & 40 & $5(12.5 \%)$ & ND & ND & & \\
\hline
\end{tabular}

Values in bold type identify those variables with a statistically significant change from ECOPD to convalescence. (Wilcoxon or Fisher exact tests, corrected for multiple comparison (false discovery rate (FDR), for continuous and categorical variables, respectively). MMRC: modified Medical Research Council; FVC: forced vital capacity; FEV1: forced expiratory volume in 1 s; IC: inspiratory capacity; RV: residual volume; TLC: total lung capacity; $D\left\llcorner C 0\right.$ : carbon monoxide diffusing capacity of the lung; $K \mathrm{CO}$ : $D\left\llcorner\mathrm{Lco/alveolar} \mathrm{volume} \mathrm{(transfer} \mathrm{factor);} \mathrm{PaO}_{2}\right.$ : arterial partial pressure of oxygen; PAFI: $\mathrm{PaO}_{2}(\mathrm{mmHg}) /$ inspired fraction of oxygen ratio (\%); $\mathrm{PaCO}_{2}$ : arterial partial pressure of carbon dioxide; 6MWD: 6-min walking distance; BNP: brain natriuretic peptide; CT: computed tomography; TAS: total antioxidant status; IL: interleukin; TNF: tumour necrosis factor; TGF: transforming growth factor; SAA: serum amyloid A; TNF-RS: tumour necrosis factor soluble receptor; RSV: respiratory syncytial virus.

convalescence was similar (51 versus 47 ), but the convalescence network was significantly denser, as shown by the higher total number of edges, a significantly higher node degree $(k)$, and lower modularity; 2 ) there were six hubs with a Kleinberg score $>0.8$ in the ECOPD network and four in the convalescence one. All of the former correspond to sputum inflammatory markers whereas all of the latter correspond to lung function variables; 3) there were five modules at ECOPD and six at convalescence (figure 2, blue areas). All of them appear relatively homogeneous in terms of their biological content, since the majority contained nodes of similar functional category (see colour codes in figure 2). A detailed description of each of these modules is provided in the supplementary material; and 4) the comparison of both networks showed a higher density of significantly different Spearman correlations at convalescence than during ECOPD (table 2 and figure 3) and that more than half of these differential correlations linked different modules (figure 3): at ECOPD, TNF- $\alpha$ was the node with more differential links $(n=4)$ whereas at convalescence these were TGF- $\beta(n=6), K C O(n=5)$, PAFI $(n=5), P a O_{2}(n=5)$ and heart rate $(n=4)$. All in all, these observations suggest that the network "perturbation" induced by ECOPD involves a reduction in module co-regulation (i.e. co-occurrence).

\section{ECOPD biomarkers: outlier analysis}

To investigate potential ECOPD biomarkers, we 1) identified 16 variables ( $12 \%$ of the total number of variables analysed in the study (table 1)) with a significant proportion of ECOPD "outliers", this is a significant (FDR p-value $<0.05$ ) proportion of variable values outside the 5th to 95th percentile range of the same variable determined at convalescence (by Monte-Carlo ECOPD/convalescence permutation test on the statistic (\% outliers at ECOPD - \% outliers at convalescence)); 2) assessed the extent to what these outliers co-occur in the same patients. To this end, we built a co-occurrence network (figure 4) where each node correspond to one of these 16 variables, node size to the proportion of outliers at ECOPD (as indicated by the percentage for each of them), node shape (up or down triangle) indicates if a given variable is higher (up) or lower (down) at ECOPD, and edge colours represent the proportion of co-occurrence between two given nodes (see keys). Circulating lymphocytes and neutrophils were co-altered (albeit in opposite directions) in more than $75 \%$ of the exacerbated patients (blue edge), and eosinophils, dyspnoea and glucose levels in $50-75 \%$ of patients (green edges; note also the different 
ECOPD

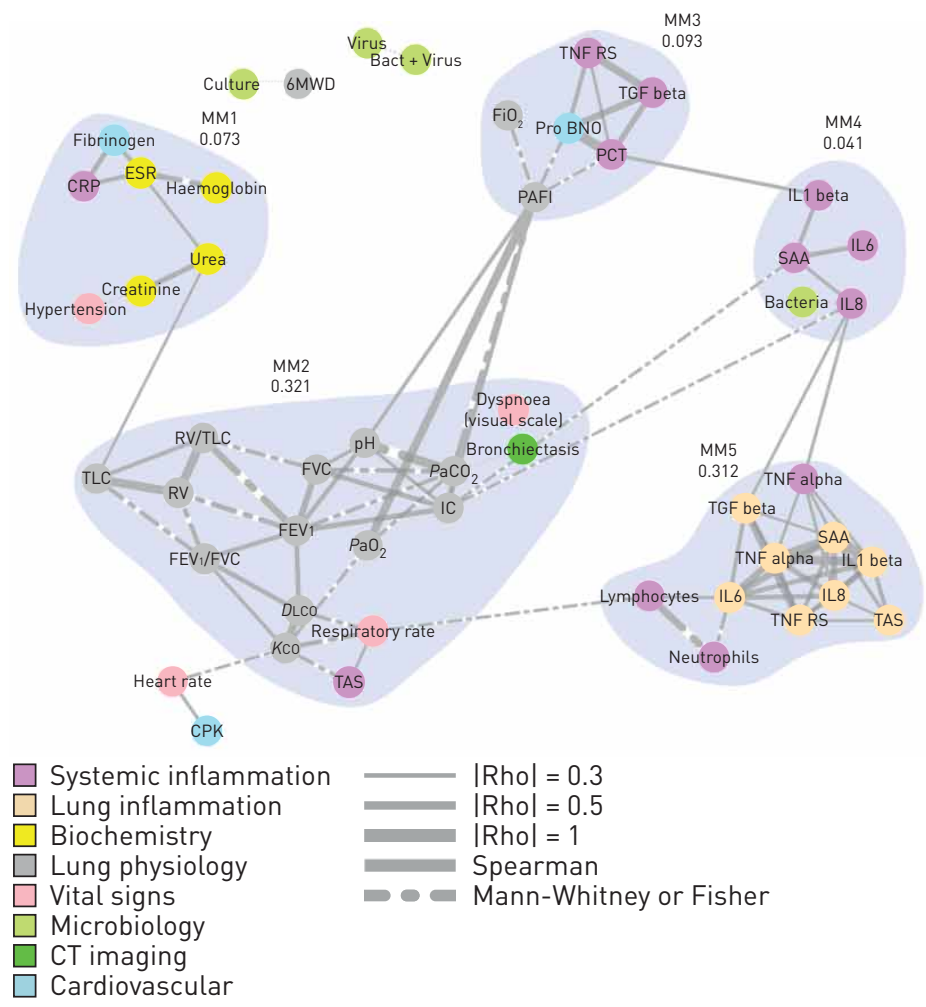

Convalescence

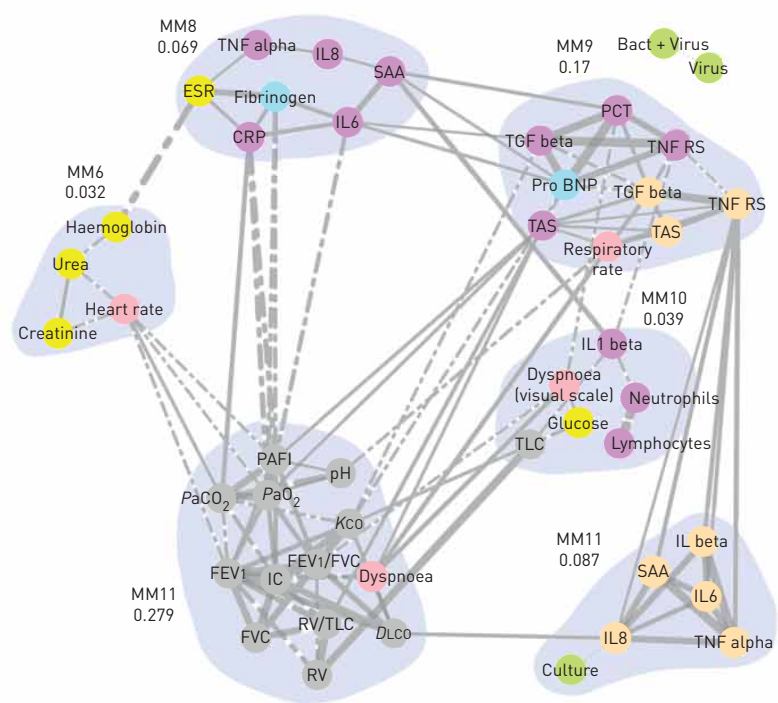

FIGURE 2 Correlation networks at exacerbation of chronic obstructive pulmonary disease (ECOPD) and convalescence. Node colours identify their category (see colour codes). Links between nodes indicate the existence of a statistically significant correlation between them (positive, continuous lines; negative, dashed lines), whereas their width is proportional to the strength of such correlation. Blue shaded areas indicate the different modules (MM) identified by the fast-greedy community algorithm used, so modules with a higher proportion of internal connections get higher MM scores. For further explanations, see text.

triangle shapes). The remaining nodes co-occurred in $25-50 \%$ of patients (orange edges); 3 ) explored the capacity of these 16 variables to predict ECOPD by ROC analysis, and identified a subset of seven of them with an area under the curve (AUC) $>0.8$. Figure 5a presents the scatter distribution of these seven variables and their individualised ROC profile and AUC (figure 5b); of note, although all of them had a large number of outliers at ECOPD (red symbols), a proportion of values at ECOPD still remained within the 5th to 95th range (horizontal lines) determined at convalescence (figure 5a), likely reflecting the heterogeneity of ECOPD episodes; and, finally, 4) included these seven variables in a general linear mixed model to identify the best diagnostic biomarker panel of ECOPD. We found that the combination of dyspnoea severity, raised circulating neutrophils and elevated CRP levels had an AUC of 0.97 (95\% CI 0.95-1) to diagnose ECOPD (figure 5c). Finally we calculated what different combinations of abnormal values of these three variables gave the better specificity, sensitivity, positive and negative prediction values for the diagnosis of an ECOPD (table 3). We observed that dyspnoea levels $\geqslant 5$ (on an analogue visual score that ranges from 0 to 10 ), CRP $\geqslant 3 \mathrm{mg} \cdot \mathrm{L}^{-1}$ and $\geqslant 70 \%$ circulating neutrophils had a specificity of 0.96 , a sensitivity of 0.901 , negative predictive value of 0.88 and positive predictive value of 0.97 for the identification of ECOPD.

\section{Discussion}

This proof-of-concept study develops and applies for the first time MLDNA to a relevant, complex and heterogeneous clinical problem (ECOPD). By doing so it shows that 1) ECOPD episodes are characterised by fragmentation of the correlation network observed during clinical stability, suggesting loss of system control and reduced resilience during ECOPD [24, 25]; and 2) a panel of biomarkers that includes dyspnoea $(\geqslant 5$ on an analogue visual score from 0 to 10$)$, CRP level $\left(\geqslant 3 \mathrm{mg} \cdot \mathrm{L}^{-1}\right)$ and $\geqslant 70 \%$ circulating neutrophils had an extremely high value (AUC 0.97) for the diagnosis of ECOPD.

\section{Previous studies}

Many studies have previously described the clinical, physiological, biological and microbiological characteristics of ECOPD [26]. By and large, our clinical observations are in keeping with them, but some 


\begin{tabular}{|c|c|c|c|}
\hline & ECOPD & Convalescence & p-value \\
\hline Number of nodes & 51 & 47 & \multirow{9}{*}{$<0.01$} \\
\hline Number of edges & 96 & 125 & \\
\hline Within-module edges/between-module edges & $12 / 84$ & $37 / 88$ & \\
\hline & TNF- $\alpha(0.92)$ & PAFI (0.97) & \\
\hline & IL-1b (0.92) & $\mathrm{PaO}_{2}(0.97)$ & \\
\hline & IL-8 (0.92) & IC $(0.90)$ & \\
\hline & TNF-RS (0.92) & & \\
\hline & IL-6 (0.88) & & \\
\hline & (all sputum variables) & & \\
\hline Within-module differential correlations/between-module differential correlations & $7 / 4$ & $24 / 19$ & $<0.01$ \\
\hline
\end{tabular}

SAA: serum amyloid A; TNF: tumour necrosis factor; IL: interleukin; TNF-RS: tumour necrosis factor soluble receptor; FEV1: forced expiratory volume in $1 \mathrm{~s} ; \mathrm{PaO}_{2}$ : arterial partial pressure of oxygen; PAFI: $\mathrm{PaO}_{2}(\mathrm{mmHg}) /$ inspired fraction of oxygen ratio (\%); IC: inspiratory capacity.

deserve specific comment. During ECOPD 1) a substantial number of patients had pulmonary hypertension and right chamber enlargement, in keeping with recent reports [27], but we did not identify patients with low ejection fraction heart failure [28]; and 2) CT found evidence of pulmonary embolism in only $1.5 \%$ of patients $[29,30]$ but, in contrast, alveolar infiltrates (not seen in chest radiographs) were identified in about a quarter of patients, as reported recently too [31]. These alveolar infiltrates can correspond to pneumonic condensations not apparent in plain chest radiographs and/or areas of local inflammation/oedema. At convalescence many (but not all) abnormalities observed during ECOPD improved. Of note, 3) even though dyspnoea and pulmonary gas exchange improved, spirometric changes only showed a statistically nonsignificant trait to improvement, which is at variance with other previous, smaller studies [32-34]; 4) as expected, several markers of systemic inflammation (total leukocyte count and levels of circulating neutrophils, CRP and fibrinogen) were reduced at convalescence. Of note, however, only $5.7 \%$ of patients showed $>2 \%$ circulating eosinophils during ECOPD, and this proportion increased up to $54.7 \%$ at convalescence. This is at variance with reports from other centres, where between $25 \%$ and $50 \%$ of the patients have $>2 \%$ circulating eosinophils during ECOPD $[3,35,36]$. We do not have a clear explanation for these discrepancies but regional differences may play a role [37]; and, finally, 5) in patients producing spontaneous sputum, the prevalence of PPM and/or viruses did not change at convalescence. Given that bronchial colonisation in clinically stable COPD patients that produce spontaneous sputum is common [38], this may have contributed to explain this lack of statistically significant changes.

\section{Interpretation of novel results \\ Homeokinetic disruptive effects of ECOPD}

Homeokinesis has been defined as "the ability of an organism to maintain a highly organised internal environment fluctuating within acceptable limits in a far from equilibrium state" [24, 25]. ECOPD episodes appear to be characterised by disrupted homeokinesis since, during clinical stability we observed a dense and well-connected correlation network with physiologically meaningful modules whereas, during ECOPD, although these modules mostly remain their connections become disrupted to a large extent (figure 2, table 2). Specifically, during clinical stability a central module (MM7), which basically includes all lung function parameters, was closely co-regulated with other modules that include pulmonary and systemic inflammatory markers (MM8, MM9, MM10) as well as a general biochemical module (MM6). By contrast, during ECOPD, the system becomes more fragmented, the sputum inflammation module (MM5) appears isolated, and systemic inflammatory markers are also less well coordinated and distributed across two different modules (MM3 and MM4). That microbiological nodes appear isolated from the main network during ECOPD probably reflects the heterogeneity of these ECOPD. Finally, the Monte Carlo 
ECOPD

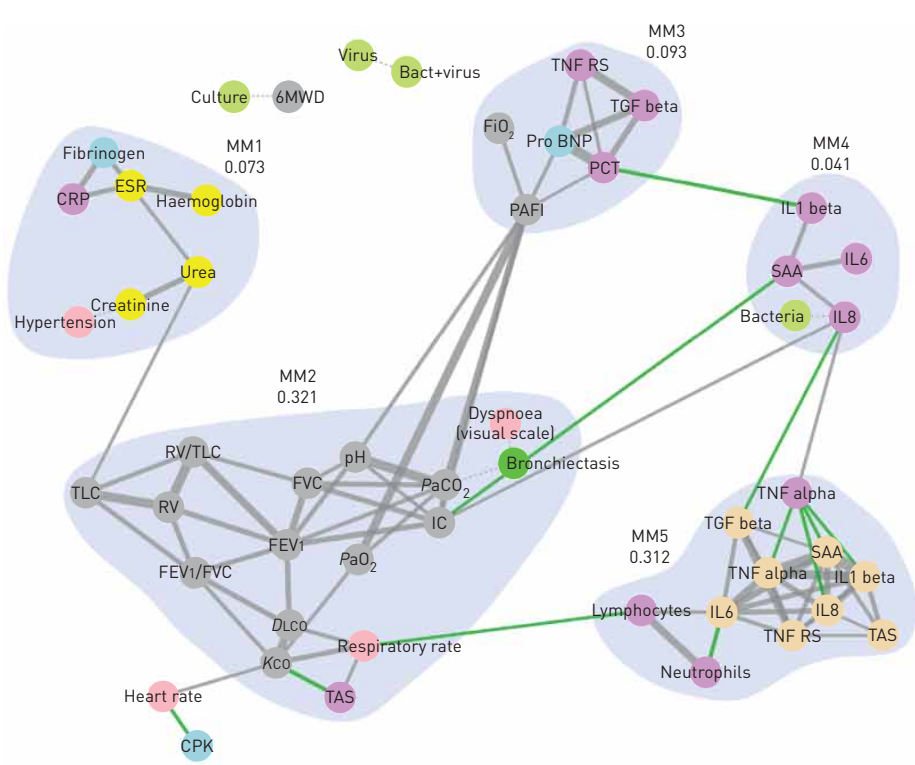

Convalescence

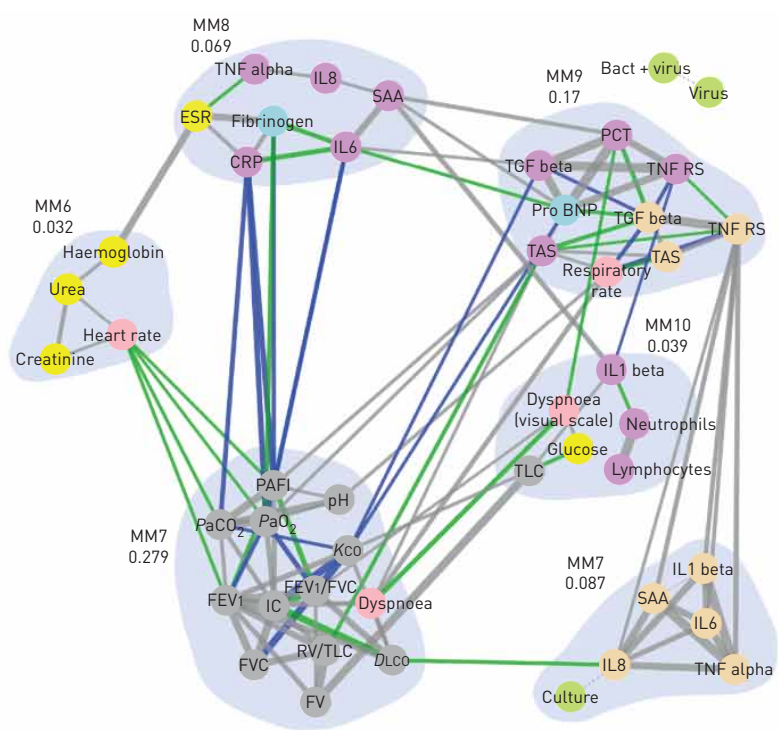

Systemic inflammation Lung inflammation

Biochemistry

Lung physiology

Vital signs

Microbiology

CT imaging

Cardiovascular

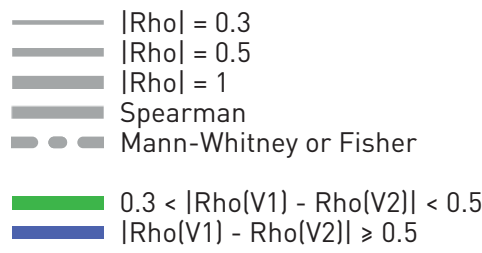

FIGURE 3 Same correlation networks presented in figure 2 now highlighting those that were significantly different at exacerbation of chronic obstructive pulmonary disease (ECOPD) and convalescence (i.e. Rho ECOPD $\neq$ Rho convalescence) by green or blue lines (see legend for Rho correspondence). For further explanations, see text.

permutation test [22] also identified more significantly different Spearman correlations at convalescence than during ECOPD (figure 3). All in all, these observations suggest that episodes of ECOPD are characterised by breakdown of the normal homeokinetic characteristics of the system with presumably less system control and resilience [24, 25].

\section{A panel of biomarkers for the diagnosis of ECOPD}

The diagnosis of ECOPD currently relies on the patient's perception of increased symptoms (mostly dyspnoea) $[1,5]$. Yet, recent research has shown that dyspnoea perception vary between patients with frequent and infrequent exacerbations [39]. Thus, having an objective way to diagnose ECOPD is of great clinical relevance [2]. Our results indicate that the combination of increased dyspnoea $(\geqslant 5)$ and raised levels of circulating neutrophils $(\geqslant 70 \%)$ and CRP $\left(\geqslant 3 \mathrm{mg} \cdot \mathrm{L}^{-1}\right)$ has an excellent value for the diagnosis of ECOPD (AUC 0.97) (figure 5c). Although the methodology we used is different, results are similar to those reported by HURST et al. [40], who showed that elevated CRP levels were the best diagnostic biomarker for ECOPD, although their diagnosis accuracy was suboptimal (AUC 0.73); however, their combination with a major exacerbation symptom (dyspnoea, sputum volume or sputum purulence) significantly increased the AUC to $0.88(\mathrm{p}<0.0001)$ [40]. Our results extend these observations further by showing that this can be further improved (AUC 0.97) by considering too the number of circulating neutrophils. The potential diagnostic utility of this biomarker panel (as well as its specific cut-off values) will have to be validated prospectively in other cohorts, but it may greatly help to advance clinical research in this area by offering for the first time an objective diagnostic tool of ECOPD. Needless to say that increased dyspnoea, elevated CRP and leukocytosis can also occur in other clinical circumstances that may not even arise from the lungs (e.g. cholecystitis, pneumonia or sickle cell crisis, among others). Therefore, the clinical context in which these three biomarkers can contribute to the diagnosis of ECOPD is of paramount importance. Finally, using unbiased cluster analysis of 182 ECOPD episodes, BAFADHEL et al. [3] recently provided convincing evidence of the heterogeneity of such episodes. Unfortunately, the relatively small sample size of our cohort $(\mathrm{n}=86)$ limits this type of analysis in our cohort. 


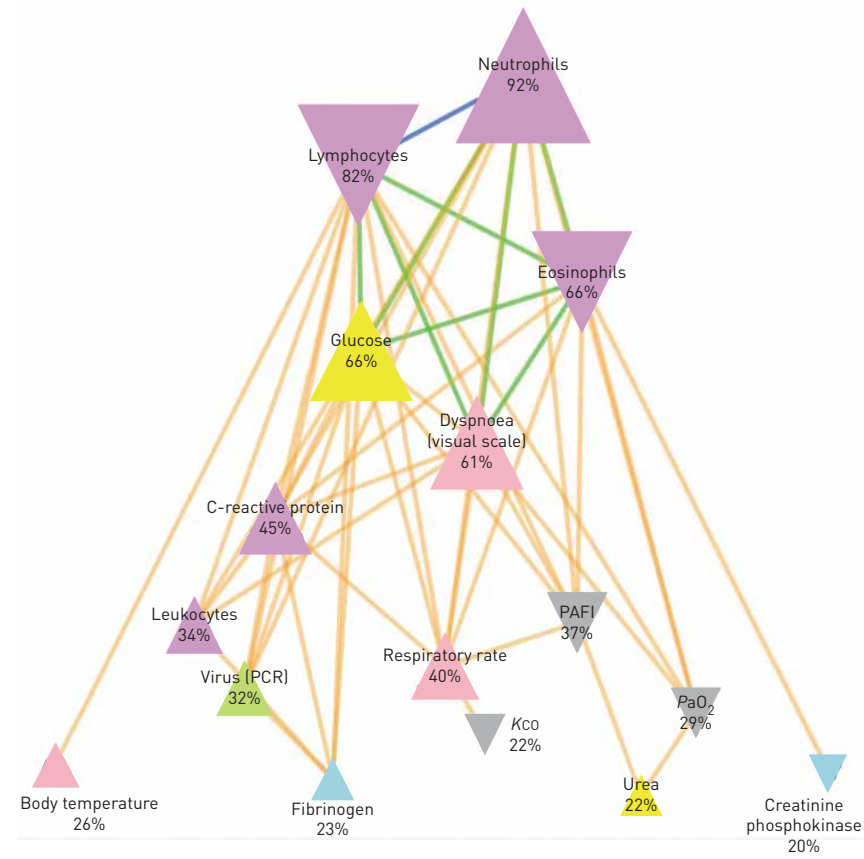

Systemic inflammation
Lung inflammation
Biochemistry
Lung physiology
Vital signs
Microbiology
CT imaging
Cardiovascular

FIGURE 4 Outlier correlation network. Nodes represent the 16 identified variables with a significant proportion of outliers at exacerbation of chronic obstructive pulmonary disease (ECOPD). Node size is proportional to the percentage of outlier values (see digits inside nodes). Edges represent shared proportions (see colour codes). The shape of the triangle (upwards or downwards) indicates that the proportion of ECOPD outliers is lower or higher than the 5th or 95th convalescence percentiles, respectively.

\section{Strengths and limitations}

The development and application, for the first time to our knowledge, of a new analytical approach (e.g. MLDNA) to get further insight into the complexity of a relevant clinical problem like ECOPD is a clear strength of our study since it provides novel, integrated, dynamic and holistic information on this frequent condition. Importantly, it also paves the way for MLDNA to be applied to other complex biological conditions in respiratory medicine and elsewhere $[6,8,16,41,42]$.

On the other hand, several potential limitations deserve comment. First, we included in the study a slightly lower number of patients $(n=86)$ than anticipated ( $n=100$; www.clinicaltrials.gov: NCT01750658), and not all measurements were available in all patients for comparison between ECOPD and convalescence. This is why we consider our study as proof-of-concept and we acknowledge that it requires validation in larger cohorts. Second, we studied severe (hospitalised) ECOPD, so our results are not directly generalisable to other milder forms of ECOPD. Third, some clinical variables, such as cough and sputum colour, were not registered. Fourth, it is not clear how much the initiation of systemic corticosteroids, before the collection of biological samples (within $72 \mathrm{~h}$ after admission) might have modified the inflammatory profile of ECOPD. Yet, it is of note that we excluded patients who received oral corticosteroid treatment before hospitalisation. Finally, patients present to hospital at various time points in the evolution of an ECOPD. All in all, we acknowledge that the results of this study will have to be confirmed in future studies, since the exclusion of severe co-morbidity, pneumonia, relatively small sample size and study of hospitalised patients (not ambulatory ECOPD) may restricts the generalisability of our observations.

\section{Conclusions}

By using a novel analytical strategy (MLDNA), this study shows that ECOPD 1) are characterised by disruption of network homeokinesis observed during clinical stability; and 2) in the appropriate clinical 

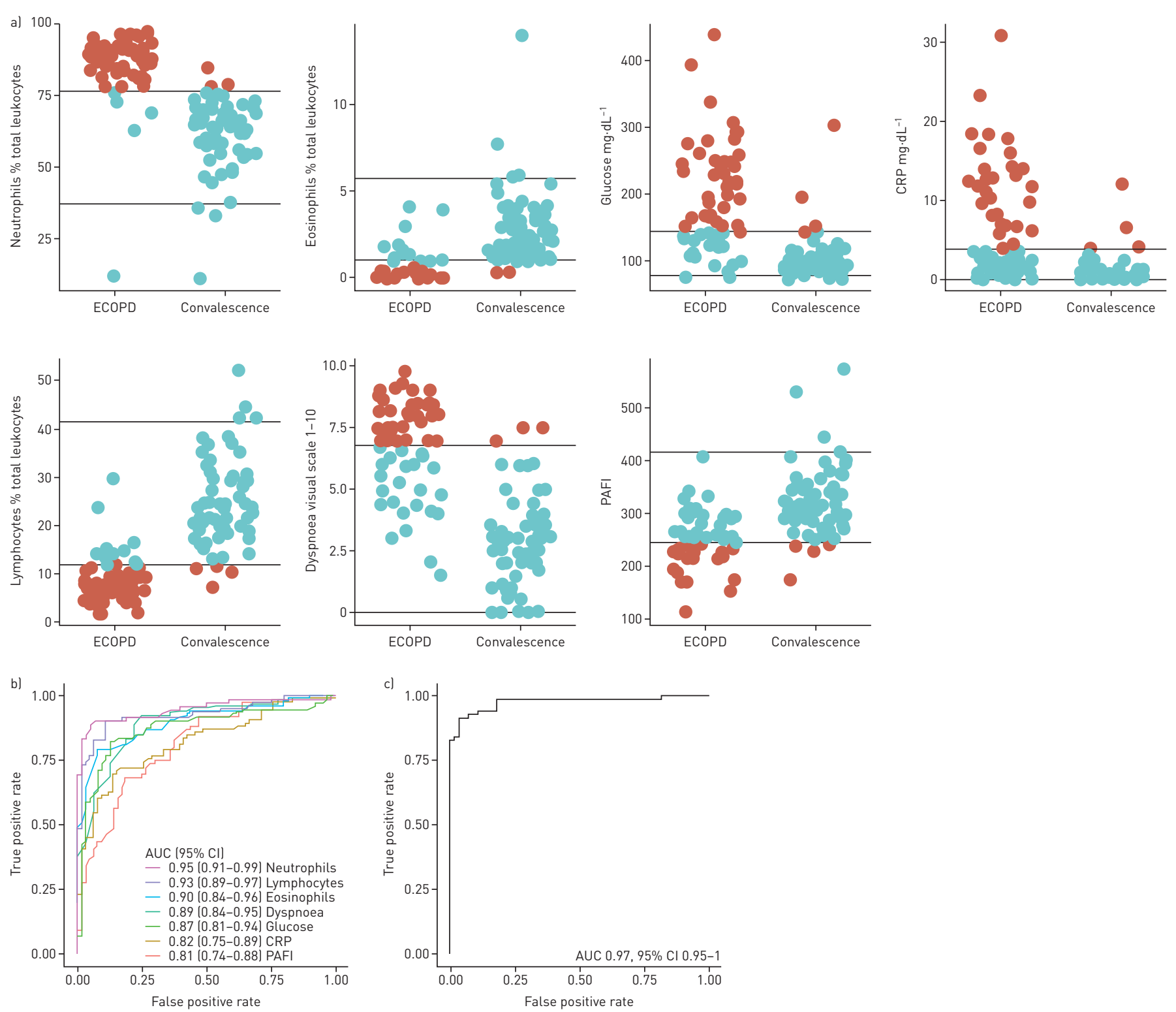

FIGURE 5 a) Scatter plot of seven continuous variables with a significant (bootstrapping FDR p-value $<0.05$ ) proportion of exacerbation of chronic obstructive pulmonary disease (ECOPD) outliers $(<5 \mathrm{TH}$ or $>95$ th percentiles (horizontal lines) at convalescence. Red symbols represent outlier values; blue symbols represent values within the convalescence 5th to 95th percentiles. b) Receiver operating characteristic curves and corresponding area under the curve (AUC) values for each of these 7 potential diagnostic biomarkers of ECOPD identified in a) with an AUC $>0.8$; c) When the seven variables identified in b) were combined in a general linear mixed model, the best panel of biomarkers to predict ECOPD (AUC 0.97) included circulating neutrophils, C-reactive protein levels and dyspnoea. For further explanations, see text.

TABLE 3 Specificity, sensitivity, negative (NPV) and positive predictive value (PPV) of a logistic regression model that includes different cut-off values of dyspnoea, C-reactive protein (CRP) and circulating neutrophil for the diagnosis of chronic obstructive pulmonary disease exacerbation (ECOPD)

\begin{tabular}{ccccccc}
$\begin{array}{c}\text { Dyspnoea } \\
\text { (visual analogue scale 1-10) }\end{array}$ & $\begin{array}{c}\text { Neutrophils } \\
\text { (\%) }\end{array}$ & $\begin{array}{c}\text { CRP } \\
\text { (mg. } \mathbf{L}^{-1} \text { ) }\end{array}$ & Specificity & Sensitivity & NPV & PPV \\
\hline \multirow{2}{*}{$\geqslant 5$} & $\geqslant 60$ & $\geqslant 3$ & 0.89 & 0.94 & 0.92 & 0.92 \\
$\geqslant 5$ & $\geqslant 65$ & $\geqslant 3$ & 0.95 & 0.91 & 0.90 & 0.95 \\
& $\geqslant 70$ & $\geqslant 3$ & 0.96 & 0.90 & 0.88 & 0.97 \\
\hline
\end{tabular}


context, ECOPD can be objectively identified by a panel of three biomarkers (dyspnoea, circulating neutrophils and CRP) commonly measured in clinical practice.

\section{Acknowledgements}

The authors thank participating patients for their willingness to contribute to medical research, and field attending physicians and nurses for their dedication and excellent care. The authors also thank Joan Soriano and Dolores Guerrero for their help in the start-up of the project, and Jørgen Vestbo, Judith Garcia-Aymerich, Josep Maria Antó and Peter J. Barnes for helpful suggestions.

\section{References}

1 Vogelmeier C, Agusti A, Anzueto A, et al. Global strategy for the diagnosis, management, and prevention of chronic obstructive pulmonary disease (2017 Report). 2017 Date last accessed: November 16, 2016.

2 Celli BR, Decramer M, Wedzicha JA, et al. An official American Thoracic Society/European Respiratory Society statement: research questions in COPD. Eur Respir J 2015; 45: 879-905.

3 Bafadhel M, McKenna S, Terry S, et al. Acute exacerbations of COPD: identification of biological clusters and their biomarkers. Am J Respir Crit Care Med 2011; 184: 662-671.

4 Beghe B, Verduri A, Roca M, et al. Exacerbation of respiratory symptoms in COPD patients may not be exacerbations of COPD. Eur Respir J 2013; 41: 993-995.

5 Rodriguez-Roisin R. Toward a consensus definition for COPD exacerbations. Chest 2000; 117: Suppl. 2, 398S-401S.

6 Barabasi AL, Gulbahce N, Loscalzo J. Network medicine: a network-based approach to human disease. Nat Rev Genet 2011; 12: 56-68.

7 Diez D, Agusti A, Wheelock CE. Network analysis in the investigation of chronic respiratory diseases: from basics to application. Am J Respir Crit Care Med 2014; 190: 981-988.

8 Menche J, Sharma A, Cho M, et al. A diVIsive Shuffling Approach (VIStA) for gene expression analysis to identify subtypes in chronic obstructive pulmonary disease. BMC Syst Biol 2014; 8: Suppl. 2, S8.

9 Rennard SI, Locantore N, Delafont B, et al. Identification of five COPD subgroups with different prognoses in the ECLIPSE cohort using cluster analysis. Ann Am Thorac Soc 2015.

10 Faner R, Gutierrez-Sacristan A, Castro-Acosta A, et al. Molecular and clinical diseasome of comorbidities in exacerbated COPD patients. Eur Respir J 2015; 46: 1001-1010.

11 Faner R, Agustí A. Network analysis: a way forward for understanding COPD multimorbidity. Eur Respir J 2015; 46: 591-592.

12 Faner R, Cruz T, López-Giraldo A, et al. Network medicine, multimorbidity and the lung in the elderly. Eur Respir J 2014; 44: 775-788.

13 Grosdidier S, Ferrer A, Faner R, et al. Network medicine analysis of COPD multimorbidities. Respir Res 2014; 15: 111.

14 Faner R, Cruz T, Casserras T, et al. Network analysis of lung transcriptomics reveals a distinct B cell signature in emphysema. Am J Respir Crit Care Med 2016; 193: 1242-1253.

15 Gustafsson M, Edstrom M, Gawel D, et al. Integrated genomic and prospective clinical studies show the importance of modular pleiotropy for disease susceptibility, diagnosis and treatment. Genome Med 2014; 6: 17.

16 Gustafsson M, Nestor C, Zhang H, et al. Modules, networks and systems medicine for understanding disease and aiding diagnosis. Genome Med 2014; 6: 82.

17 Benjamini Y, Hochberg Y. Controlling the false discovery rate: a practical and powerful approach to multiple testing. J R Stat Soc Ser B (Methodological) 1995; 57: 289-300.

18 R Core Team. R: A language and environment for statistical computing. R Foundation for Statistical Computing, Vienna, Austria, 2015

19 Shannon P, Markiel A, Ozier O, et al. Cytoscape: a software environment for integrated models of biomolecular interaction networks. Genome Res 2003; 13: 2498-2504.

20 Newman ME, Girvan M. Finding and evaluating community structure in networks. Phys Rev E Stat Nonlin Soft Matter Phys 2004; 69: 026113.

21 Su G, Kuchinsky A, Morris JH, et al. GLay: community structure analysis of biological networks. Bioinformatics 2010; 26: 3135-3137.

22 Fishman GS. Monte Carlo: concepts, algorithms and applications. New York, Springer, 1995.

23 Davies L, Angus RM, Calverley PM. Oral corticosteroids in patients admitted to hospital with exacerbations of chronic obstructive pulmonary disease: a prospective randomised controlled trial. Lancet 1999; 354: 456-460.

24 Macklem PT. Emergent phenomena and the secrets of life. J Appl Physiol 2008; 104: 1844-1846.

25 Macklem PT, Seely A. Towards a definition of life. Perspect Biol Med 2010; 53: 330-340.

26 Wedzicha JA, Singh R, Mackay AJ. Acute COPD exacerbations. Clin Chest Med 2014; 35: 157-163.

27 Freixa X, Portillo K, Pare C, et al. Echocardiographic abnormalities in patients with COPD at their first hospital admission. Eur Respir J 2013; 41: 784-791.

28 Singanayagam A, Schembri S, Chalmers JD. Predictors of mortality in hospitalized adults with acute exacerbation of chronic obstructive pulmonary disease. Ann Am Thorac Soc 2013; 10: 81-89.

29 Roca M, Verduri A, Corbetta L, et al. Mechanisms of acute exacerbation of respiratory symptoms in chronic obstructive pulmonary disease. Eur J Clin Invest 2013; 43: 510-521.

30 Tillie-Leblond I, Marquette CH, Perez T, et al. Pulmonary embolism in patients with unexplained exacerbation of chronic obstructive pulmonary disease: prevalence and risk factors. Ann Intern Med 2006; 144: 390-396.

31 Cheng $\mathrm{T}$, Wan $\mathrm{H}$, Cheng Q, et al. Computed tomography manifestation of acute exacerbation of chronic obstructive pulmonary disease: A pilot study. Exp Ther Med 2016; 11: 519-529.

32 Stevenson NJ, Walker PP, Costello RW, et al. Lung mechanics and dyspnea during exacerbations of chronic obstructive pulmonary disease. Am J Respir Crit Care Med 2005; 172: 1510-1516.

33 Parker CM, Voduc N, Aaron SD, et al. Physiological changes during symptom recovery from moderate exacerbations of COPD. Eur Respir J 2005; 26: 420-428. 
34 Cote CG, Dordelly LJ, Celli BR. Impact of COPD exacerbations on patient-centered outcomes. Chest 2007; 131: 696-704.

35 Bafadhel M, McKenna S, Terry S, et al. Blood eosinophils to direct corticosteroid treatment of exacerbations of chronic obstructive pulmonary disease: a randomized placebo-controlled trial. Am J Respir Crit Care Med 2012; 186: 48-55.

36 Bafadhel M, Davies L, Calverley PMA, et al. Blood eosinophil guided prednisolone therapy for exacerbations of COPD: a further analysis. Eur Respir J 2014; 44: 789-791.

37 Saha S, Brightling CE. Eosinophilic airway inflammation in COPD. Int J Chron Obstruct Pulmon Dis 2006; 1: 39-47.

38 Bandi V, Apicella MA, Mason E, et al. Nontypeable Haemophilus influenzae in the lower respiratory tract of patients with chronic bronchitis. Am J Respir Crit Care Med 2001; 164: 2114-2119.

39 Scioscia G, Blanco I, Burgos F, et al. Different dyspnea perception in COPD patients with frequent and infrequent exacerbations. Thorax 2017; 72: 117-121.

40 Hurst JR, Donaldson GC, Perera WR, et al. Use of plasma biomarkers at exacerbation of chronic obstructive pulmonary disease. Am J Respir Crit Care Med 2006; 174: 867-874.

41 Menche J, Sharma A, Kitsak M, et al. Disease networks. Uncovering disease-disease relationships through the incomplete interactome. Science 2015; 347: 1257601.

42 Ghiassian SD, Menche J, Barabasi AL. A DIseAse MOdule Detection (DIAMOnD) algorithm derived from a systematic analysis of connectivity patterns of disease proteins in the human interactome. PLoS Comput Biol 2015; 11: e1004120. 